\title{
Perivascular epithelioid cell tumor of the descending colon mimicking a gastrointestinal stromal tumor: a case report
}

\author{
Ryuta Iwamoto ${ }^{1,2}$, Tatsuki R. Kataoka ${ }^{1 *}$, Ayako Furuhata ${ }^{1}$, Kazuo Ono ${ }^{2}$, Seiichi Hirota ${ }^{3}$, Kenji Kawada ${ }^{4}$, \\ Yoshiharu Sakai ${ }^{4}$ and Hironori Haga ${ }^{1}$
}

\begin{abstract}
Background: We present a case of perivascular epithelioid cell tumor (PEComa), which clinically and histologically mimics a gastrointestinal stromal tumor (GIST).

Case presentation: A 42-year-old woman was found to have a mass in the left flank during her annual medical checkup. Computed tomography examination revealed a submucosal tumor of the descending colon. Surgeons and radiologists suspected that the lesion was a GIST, and left hemicolectomy was performed without biopsy. Microscopic examination showed that the lesion was composed of spindle and epithelioid cells, which were immunohistochemically negative for c-kit and positive for platelet-derived growth factor receptor (PDGFR) a. Initial diagnosis of PDGFRa-positive GIST was made. However, gene analysis did not reveal mutations in PDGFRa. Additional immunohistochemistry showed that tumor cells were positive for human melanin black 45 (HMB45), melanA, and the myogenic marker calponin. A final diagnosis of PEComa was made.
\end{abstract}

Conclusion: PEComa should be included in the differential diagnosis of PDGFRa-positive spindle cell tumors in the wall of the gastrointestinal tract.

Keywords: Gastrointestinal stromal tumor, KIT, Perivascular epithelioid cell tumor, Platelet-derived growth factor receptor

\section{Background}

Gastrointestinal stromal tumor (GIST) is the most common mesenchymal tumor in the walls of the gastrointestinal tract [1]. GISTs typically harbor gain-of-function type mutations in the KIT genes [2], and GISTs without KIT mutations have gain-of-function type mutations in the platelet-derived growth factor receptor (PDGFR) $\alpha$ genes [3]. Expression of the two genes is mutually exclusive [1-3]. Perivascular epithelioid cell tumor (PEComa) is a less common mesenchymal tumor, expressing melanocytic and myogenic markers such as actin, desmin, calponin, human melanin black (HMB) 45, melanA, and microphthalmia-associated transcription factor (MITF)

\footnotetext{
* Correspondence: trkataoka@yahoo.co.jp; trkata@kuhp.kyoto-u.ac.jp ${ }^{1}$ Department of Diagnostic Pathology, Kyoto University Hospital, 54 Syogoinkawahara-cho, Sakyo-ku, Kyoto 606-8507, Japan

Full list of author information is available at the end of the article

[4]. PEComa can occur in any organs, but is rarely detected in the gastrointestinal wall [5]. Herein, we report a case of PDGFR $\alpha$-positive PEComa arising in the wall of the descending colon.

\section{Case presentation}

A 42-year-old woman underwent abdominal ultrasonography during her annual medical checkup and a mass in her left flank region was identified. She was admitted to the hospital for further examination. A computed tomography scan and endoscopic examination revealed a submucosal tumor in the wall of the descending colon. Systemic magnetic resonance imaging and positron emission tomography scans did not show any other lesions. The lesion was suspected to be a colonic GIST and left hemicolectomy was performed. Upon macroscopic examination, the tumor was $5 \mathrm{~cm}$ in the greatest 


\section{A Gross}

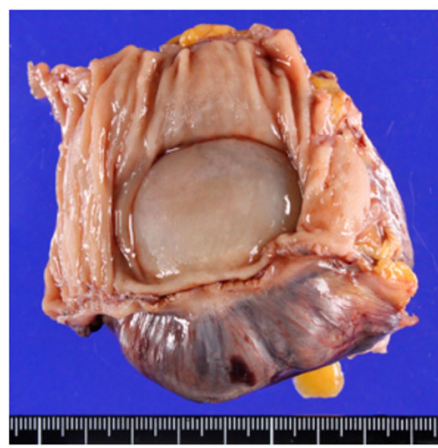

B Sliced

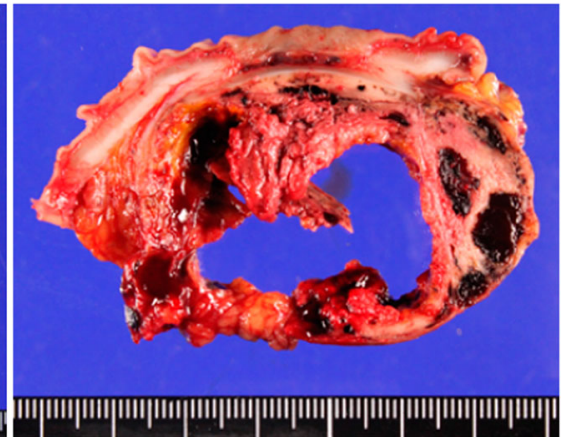

Fig. 1 Macroscopic findings. a Gross appearance. b Sliced specimens

dimension, well-circumscribed but uncapsulated, and extended from the muscular propria into the subserosa (Fig. 1a). The cut surface was hemorrhagic and necrotic (Fig. 1b). Microscopically, the tumor cells consisted of spindle and epithelioid cells with a granular cytoplasm (Fig. 2a). Based on the clinical diagnosis of GIST, a panel of immunohistochemistry including KIT, PDGFR $\alpha$, discovered on GIST-1 (DOG1), CD34, S100, desmin, and Ki67 were performed. The tumor cells were positive for PDGFR $\alpha$ (Fig. 2b) and negative for KIT (Fig. 2c), DOG1
(Fig. 2d), CD34, S100, and desmin. The Ki-67 index was 3\% (Fig. 2e). We initially suspected the tumor to be a PDGFR $\alpha$-positive GIST. Mutational analysis did not reveal any mutation in PDGFR $\alpha$ or KIT, and suggested the possibility of a low-grade tumor other than GIST. Upon further examination, the tumor cells were found to be positive for HMB45 (Fig. 2f) and calponin (Fig. 2g), and negative for melanA, MITF, SOX10, and actin. These results were compatible with PEComa. This tumor was immunohistochemically negative for
A H\&E

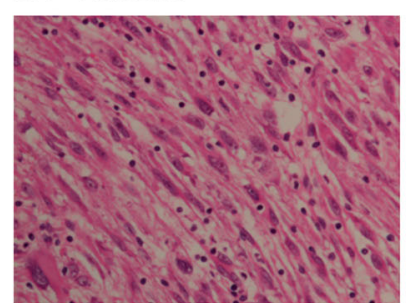

C KIT

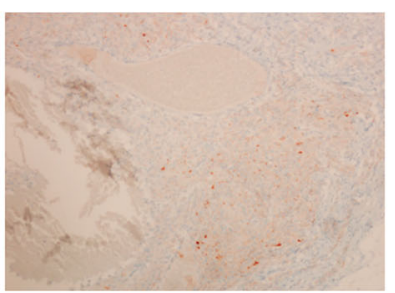

F HMB45

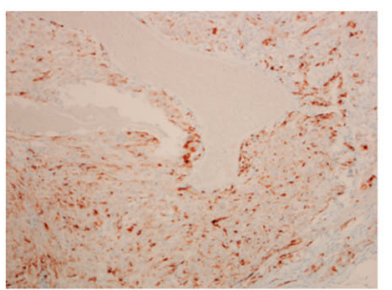

\section{B PDGFR $\alpha$}

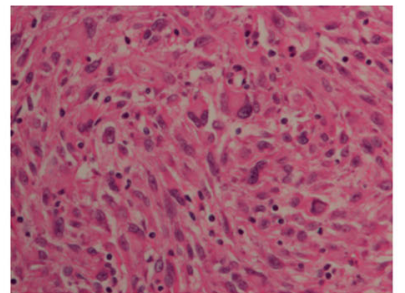

D DOG1

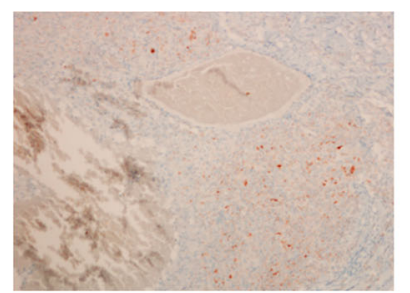

\section{G Calponin}

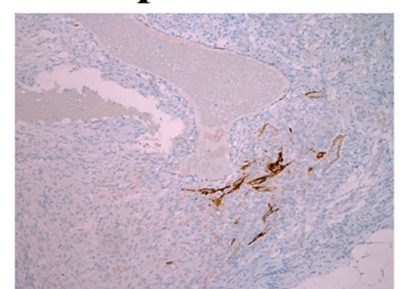

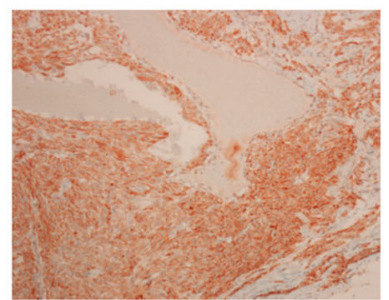

E Ki67

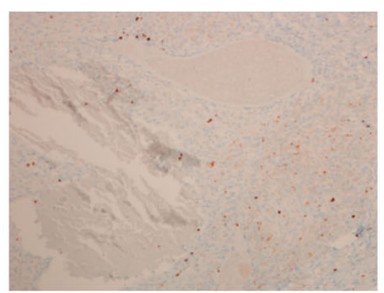

H TFE3

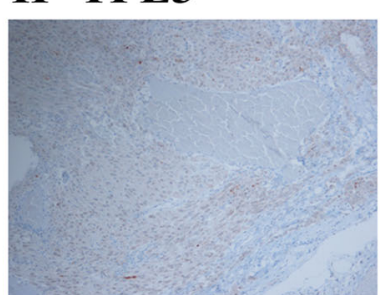

Fig. 2 Histological findings. a Hematoxylin and eosin (H\&E) staining. Two representative fields. Immunohistochemical specimens for $\mathbf{b}$ PDGFRa, $\mathbf{c}$ KIT, $\mathbf{d}$ discovered on GIST-1 (DOG1), e Ki67, f HMB45, g Calponin, and $\mathbf{h}$ TFE3. Photos are $\times 200$ magnification in $\mathbf{a}$ and $\times 100$ magnification in $\mathbf{b}-\mathbf{h}$ 
TFE3 (Fig. 2h), but did not show rearrangement of TFE3 in fluorescence in situ hybridization (FISH) (data not shown). The patient was alive without recurrence 5 months after the resection.

\section{Discussion}

PEComa is rare in the gastrointestinal tract. To the best of our knowledge, only 36 cases of gastrointestinal PEComa have been reported sporadically [6, 7]. Doyle et al. performed a clinicopathologic study of 35 cases of gastrointestinal PEComa [5]. The current case shows similarities with previously reported cases of gastrointestinal PEComa, in terms of the clinicopathological features and immunological profile. GIST does not show immunoreactivity for melanocytic markers [8], and expression of HMB45 is important to support the diagnosis of PEComa. Metastatic melanoma is positive for HMB45, but is also positive for S100 protein and lacks expression of myogenic markers such as calponin. Some cases of PEComa show gene rearrangement involving TFE3, and strong nuclear TFE3 expression $[4,5]$. In our case, TFE3 rearrangement was not detected by FISH. This result is not incompatible with a diagnosis of PEComa because most gastrointestinal PEComas are negative for TFE3 [5]. Thus, TFE3 status may not be a diagnostic clue in gastrointestinal PEComa.

The tumor cells in our case were partly epithelioid and immunohistochemically PDGFR $\alpha$-positive. These phenotypes were thought to be compatible with the initial diagnosis of GISTs with a PDGFR $\alpha$ mutation [3, 9]. However, GISTs with a PDGFR $\alpha$ mutation most commonly arise in the stomach $[9,10]$, and the tumor is typically DOG1-positive [11, 12]. In contrast to the case of GISTs, PDGFR $\alpha$ positivity and mutations in PDGFR $\alpha$ genes have not been reported in PEComas, to the best of our knowledge. The status of PDGFR $\alpha$ in PEComa should be further studied to diagnose PDGFR $\alpha$-positive mesenchymal tumors in the gastrointestinal tract. Both GISTs and PEComas are treated with surgical resection and chemotherapy. GISTs are susceptible to the tyrosine kinase inhibitor imatinib [10], although PEComas are susceptible to another inhibitor, sirolimus [13]. Therefore, distinguishing between GISTs and PEComas would be important for appropriate administration of kinase inhibitors.

\section{Conclusion}

PEComa should be included in the differential diagnosis of mesenchymal tumors in the wall of the gastrointestine, even though tumor cells are immunohistochemically PDGFR $\alpha$-positive. Mutational analysis should be performed to confirm the diagnosis of GIST, even though PDGFR $\alpha$ is immunohistochemically positive.

\section{Abbreviations}

DOG1: Discovered on GIST-1; GIST: Gastrointestinal stromal tumor; HMB45: Human melanin black 45; MITF: Microphthalmia-associated transcription factor; PDGFR: Platelet-derived growth factor receptor; PEComa: Perivascular epithelioid cell tumor; TFE3: Transcriptional factor E3

\section{Acknowledgments}

The authors thank Ms. ljiri K (Department of Diagnostic Pathology, Kyoto University Hospital, Kyoto, Japan) for her secretarial assistance.

Funding

TRK was supported by a grant from the Ministry of Education, Culture, Sports, Science and Technology, Japan (15K08362).

\section{Availability of data and materials}

All data on which the conclusions of this case report are included in this manuscript.

\section{Authors' contributions}

$\mathrm{RI}, \mathrm{TRK}, \mathrm{AF}, \mathrm{KO}, \mathrm{SH}$, and $\mathrm{HH}$ were all involved in this report's conception and coordination, and helped to draft the manuscript. Additionally, all authors read and approved the final version of the manuscript.

\section{Competing interests}

The authors declare that they have no competing interests.

\section{Consent for publication}

The patient signed the Kyoto University Hospital Informed Consent Form for the Non-therapeutic Use of Histopathological Materials, and the signed forms have been uploaded into her electronic health record.

Ethics approval and consent to participate

This case report does not require ethical approval.

\section{Author details}

'Department of Diagnostic Pathology, Kyoto University Hospital, 54 Syogoinkawahara-cho, Sakyo-ku, Kyoto 606-8507, Japan. ${ }^{2}$ Department of Pathology, Japan Red Cross Society Wakayama Medical Center, 4-20 Komatsubara-dori, Wakayama 640-8558, Japan. ${ }^{3}$ Department of Surgical Pathology, Hyogo College of Medicine, 1-1 Mukogawa-cho, Nishinomiya, Hyogo 663-8501, Japan. ${ }^{4}$ Department of Surgery, Kyoto University Hospital, 54 Syogoinkawahara-cho, Sakyo-ku, Kyoto 606-8507, Japan.

Received: 27 July 2016 Accepted: 7 November 2016

Published online: 14 November 2016

References

1. Hirota S, Isozaki K. Pathology of gastrointestinal stromal tumors. Pathol Int. 2006:56:1-9.

2. Hirota S, Isozaki K, Moriyama Y, Hashimoto K, Nishida T, Ishiguro S, Kawano K, Hanada M, Kurata A, et al. Gain-of-function mutations of c-kit in human gastrointestinal stromal tumors. Science. 1998;279:577-80.

3. Hirota S, Ohashi A, Nishida T, Isozaki K, Kinoshita K, Shinomura Y, Kitamura Y Gain-of-function mutations of platelet-derived growth factor receptor alpha gene in gastrointestinal stromal tumors. Gastroenterology. 2003;125:660-7.

4. Martignoni G, Pea M, Reghellin D, Zamboni G, Bonetti F. PEComas: the past, the present and the future. Virchows Arch. 2008:452:119-32.

5. Doyle LA, Hornick JL, Fletcher CD. PEComa of the gastrointestinal tract: clinicopathologic study of 35 cases with evaluation of prognostic parameters. Am J Surg Pathol. 2013;37:1769-82.

6. Medeiros F, Corless CL, Duensing A, Hornick JL, Oliveira AM, Heinrich MC, Fletcher JA, Fletcher CD. KIT-negative gastrointestinal stromal tumors: proof of concept and therapeutic implications. Am J Surg Pathol. 2014;28:889-94.

7. Liegl-Atzwanger B, Fletcher JA, Fletcher CD. Gastrointestinal stromal tumors. Virchows Arch. 2010;456:111-27.

8. West RB, Corless CL, Chen X, Rubin BP, Subramanian S, Montgomery K, Zhu S, Ball CA, Nielsen TO, et al. The novel marker, DOG1, is expressed ubiquitously in gastrointestinal stromal tumors irrespective of KIT or PDGFRA mutation status. Am J Pathol. 2014;165:107-13. 
9. Miettinen M, Wang ZF, Lasota J. DOG1 antibody in the differential diagnosis of gastrointestinal stromal tumors: a study of 1840 cases. Am J Surg Pathol. 2009;33:1401-8

10. Chen Z, Shi H, Peng J, Yuan Y, Chen J, Song W. Perivascular epithelioid cell tumor in the duodenum: challenge in differential diagnosis. Int J Clin Exp Pathol. 2015:8:8555-62.

11. Lu B, Wang C, Zhang J, Kuiper RP, Song M, Zhang X, Song S, van Kessel AG, Iwamoto A, et al. Perivascular epithelioid cell tumor of gastrointestinal tract: case report and review of the literature. Medicine (Baltimore). 2015;94:e393.

12. Wong NA, Melegh Z. Gastrointestinal stromal tumours can express CD10 and epithelial membrane antigen but not oestrogen receptor or HMB45. Histopathology. 2011;59:781-5.

13. Bissler JJ, McCormack FX, Young LR, Elwing JM, Chuck G, Leonard JM, Schmithorst VJ, Laor T, Brody AS, et al. Sirolimus for angiomyolipoma in tuberous sclerosis complex or lymphangioleiomyomatosis. N Engl J Med. 2008;358:140-51.

Submit your next manuscript to BioMed Central and we will help you at every step:

- We accept pre-submission inquiries

- Our selector tool helps you to find the most relevant journal

- We provide round the clock customer support

- Convenient online submission

- Thorough peer review

- Inclusion in PubMed and all major indexing services

- Maximum visibility for your research

Submit your manuscript at www.biomedcentral.com/submit
Biomed Central 